\title{
The Effects of Ammonia Inhalants on Anaerobic Performance Following a Simulated American Football Game
}

\author{
Secrest $\mathrm{JR}^{1,2}$, Jones $\mathrm{EJ}^{2}$, Faries $\mathrm{MD}^{2}$, Hearon $\mathrm{CM}^{1}$ \\ ${ }^{1}$ Department of Health and Kinesiology; Texas A\&M University-Kingsville; Kingsville, \\ TX
}

2Department of Kinesiology and Health Science; Stephen F. Austin State University; Nacogdoches, TX
Category: Masters
Advisor / Mentor: Jones, Eric (jonesej@sfasu.edu)
ABSTRACT

PURPOSE: Ammonia inhalants (AI) are anecdotally referred to as ergogenic aids in the realm of aggressive team/lifting sports. However, the efficacy of AI usage has yet to be established or reported to our knowledge. Therefore, the purpose of this study was to establish the effects of AI's on anaerobic performance following a simulated American football game (SAFG) in a climate controlled setting (35.8 $\left.{ }^{\circ} \mathrm{C}, 27.5 \% \mathrm{RH}\right)$. METHODS: Ten apparently healthy anaerobically trained college-aged males participated dressed in American football apparel, excluding the helmet. Each subject participated in two trials, 136 minutes each, separated by a minimum of 48 hours (AI or control). Each SAFG consisted of a randomized counter-balanced series of sprints. The sprint protocol was made up of a total 12, 9, or 6 sprints, which were experienced each half of the SAFG in a randomized order. The individual sprints consisted of 5 second maximal effort sprints which were followed by 40 seconds of rest, simulating what one would expect in an American football game. The Wingate anaerobic test (WAnT) was performed before and after each SAFG in a neutral temperature $\left(21.3^{\circ} \mathrm{C}, 30.8 \% \mathrm{RH}\right)$. Resistance for the 30 -second WAnT was calculated at $10 \%$ of each respective subject's weight in kilograms $(\mathrm{kg})$. The AI condition was administered by capsule immediately before the final WAnT. Paired $t$-tests were used to examine differences in peak power change $(\triangle \mathrm{PP})$ and mean power change $(\triangle \mathrm{MP})$ from WAnTs performed pre-post-SAFG. $\mathrm{a}=0.05$. RESULTS: The majority of our subjects elicited an increase in PP ( $9 / 10$ subjects) and MP (8/10 subjects) during the $\mathrm{AI}$ condition. The $\triangle \mathrm{PP}$ in the $\mathrm{AI}(134 \pm 85 \mathrm{~W})$ trial was significantly greater that the control trial $(59 \pm 108 \mathrm{~W})(p \leq .05)$. Likewise, $\triangle \mathrm{MP}$ in the $\mathrm{AI}(35 \pm 53 \mathrm{~W})$ was significantly greater than the control $(-21 \pm 65$ $\mathrm{W})$ trial $(p \leq .01)$. CONCLUSION: These results suggest that the intervention of AI following a bout of fatiguing work is successful in the in the generation of maximal power outputs as well as attenuating fatigue within the WAnT. Thereby, suggesting that when performing a WAnT, utilizing AI as an intervention can increase power output. Further research should be conducted in order to support the findings from the present investigation and possible applications. 